\title{
Comparison between endocrown fracture resistance with post core crown
}

\author{
${ }^{1}$ Yohanes Paulus Krisna, ${ }^{2}$ Ira Tanti \\ ${ }^{1}$ Prosthodontic Residency Program \\ ${ }^{2}$ Prosthodontic Department \\ Faculty of Dentistry, Indonesia University, \\ Jakarta, Indonesia. \\ Corresponding author: Ira Tanti, Email: iratanti@ymail.com
}

\begin{abstract}
The concept of minimally invasive restoration has been more approached lately, with the aim of preserve the remaining tooth structure especially after endodotic treatment. Endocrown is one type of restoration that might be the solution. Endocrown is made of glass-ceramic material, defined as a single restoration that uses pulp chambers as a retention and resistance form, margin of preparation is above the gingiva in order to preserve tooth structure. Endocrown was initiated by Pissis in 1995 and popularized by Bindl and Mörmann in 1999. According to late research, crown endocrown has higher fracture resistance than post-core crown. Although the literature on endocrown is still limited, empirical evidence leads to positive view for the use of this restoration.
\end{abstract}

Keywords: endocrown, fracture resistance, postcore

\section{INTRODUCTION}

Dental rehabilitation after endodontic treatment which has extensive caries damage is still a challenge. First, most of the tooth structure has been lost, so the rest of the structure provides minimal retention and resistance form. Second, even though the remaining tissue structure is minimal, sometimes additional preparations are still needed to form margins for the restoration material. Third, the use of metal post or fiber post can weaken the root structure because of the preparation of a rootcanal widening. These three things become the dentist's dilemmain making decisions.

Elderton and Simonsen state when a tooth receives a restoration, it enters a 'restorative cycle of death'. ${ }^{1}$ A restoration will be defective at sometime will be replaced by another bigger restoration, and finally the tooth will be extracted. The concept of minimally invasive restoration has been moreapproached lately, with the aim of preserving the remaining tooth structure, especially after endodontic treatment. Meanwhile, according to in-vitro and in-vivo studies, post was not proven to have a significant effecton the success of longterm treatment of tooth after endodontic treatment. It weakens the structure of the remaining tooth through preparation and enlargement of the root canal. ${ }^{2,3}$ Alternative restoration treatment which is more minimally invasive, minimal preparation and preserves the remaining tooth structure is needed. One of the alternative restoration solutions is a crown with internal extension into pulp chamber, and is known by the name endocrown. ${ }^{4}$

Endocrown restoration was first introduced by Pissis in 1995 under the name monoblock porcelain technique. The term endocrown (endodontic adhesive crown) itself only began in 1999 by Bindl and Mörmann. ${ }^{5}$ A characteristic of endocrown using porcelain crowns that cover the entire tooth surface is by extending the internal side of the restoration into the pulp chamber. Its macromechanical retention is obtained from the pulp wall and the micromechanical retention is obtained through adhesive cement. The stress distribution on the endocrown is more evenly distributed compared to the post-core crown, because the endocrown crown is a single unit. However this will be greatly influenced by the type of crown material used. ${ }^{6}$ The use of endocrown to date is still being debated. Although the evidence of clinical studies leads to the positive side, its empirical evidence is less than the use of post-core crown. Therefore the use of endocrown as restoration is still on the decision of the clinician who work on it. In this article, this paper aims to compare whether endocrown is better than a post core crown in fracture resistance.

\section{METHOD}

This paper is compiled based on the problems, interventions, comparisons, results (PICO) model, whether tooth with root canal treatment (P) restored withendocrown (I)compared postand crown $(\mathrm{C})$ has better fracture resistance $(\mathrm{O})$ ?

Data collection were screened on March 31, 2020 from Pubmed, EBSCO and Scopus using the search strategy as follow: ((endocrown) AND ((zirconia post) OR (metal post) OR (cast post) OR (fiber post))) AND ((fracture resistance) OR (strength)). Data were extracted into csv. files from 
each search engine combined into the same sheetin Excel 365 (Microsooft Corporation, Redmond, WA, USA). Duplicate were eliminated by sorting the same DOI. This article exclusion will be carried out twice, the first based on the title and abstract and the second exclusion is based on the methodology (Fig. 1).

\section{RESULTS}

A total of 55 relevant articles were identified and 13 of these are duplicate. The remaining 42 articles are examined based on title and abstract, 10 studies were excluded because they did not meet the eligibility criteria, 25 more articles were exluded based on method. Thus, 7 remaining articles will be discussed in this paper (Table 1).

Each remaining articles are in-vitro studies which investigating fracture strength and were published between 2015 and 2020 . The sample size ranged from 30 to 105 teeth by study. Two studies analyzed endocrowns in anterior teeth, while five studies in posterior teeth. All studies evaluated lithium disilicate endocrown and fiber post with lithium disilicate restoration, except two studies, one using composites rather than ceramics for crown restoration combined with fiber post and another use glass-ceramic combined with fiber post (Table 2). Load testing methods between studies are varies and some of them are not decribed in full. Two studies did not write the standard deviation results.

The result within studies are varies. There is no uniformity among the study states that tooth with endocrown is better than post and crown, including the type of fracture that occurs (Table 3). Favorable fracture is a type of fracture where the tooth can still be considered for restoration, while unfavorable fracture is the contrary (more than cementoenameljunction). However, most of the studies states endocrown has better fracture resistance than post and crown. ${ }^{7-10}$

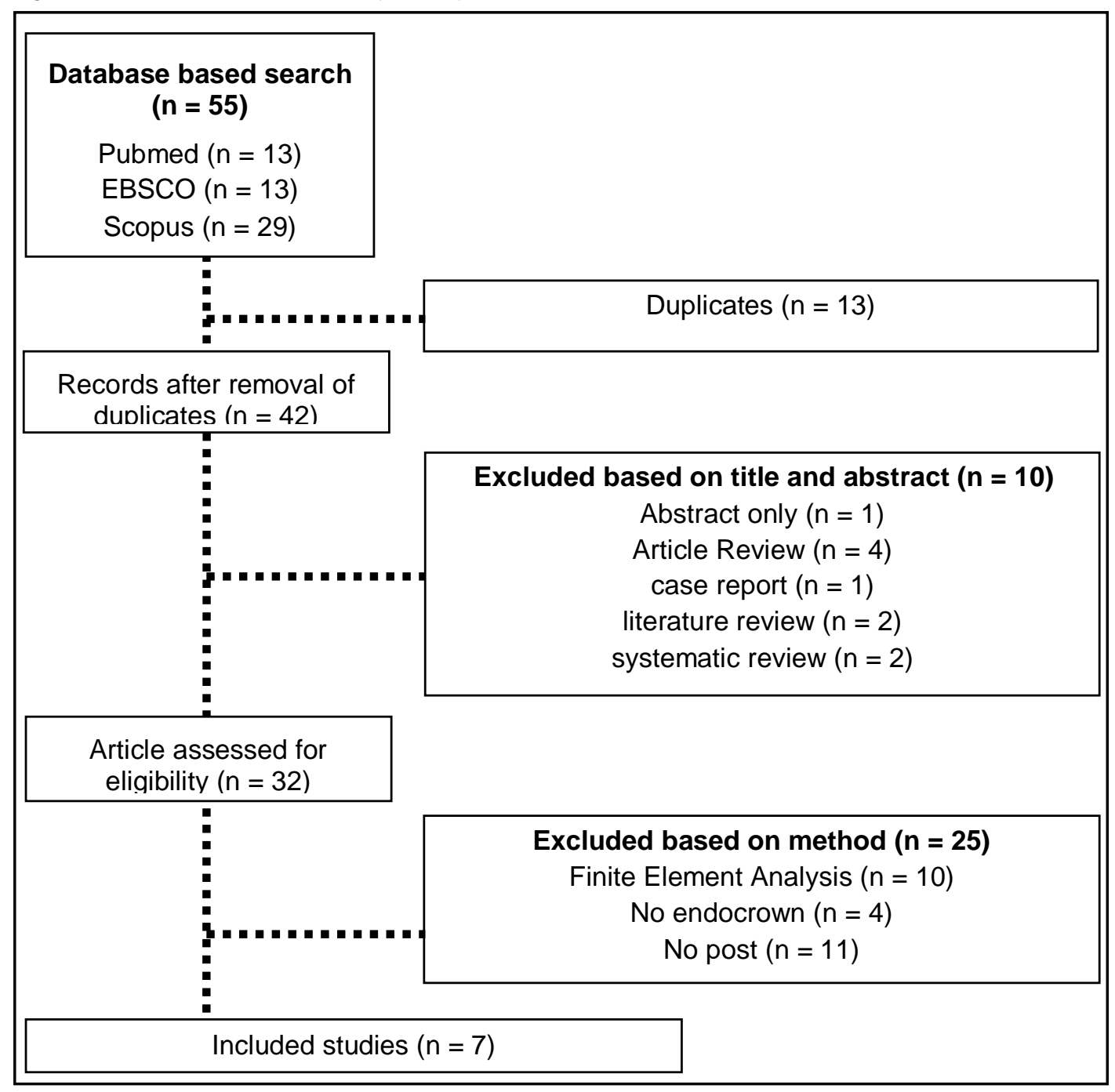

Fig. 1 Data collection workflow 
Tabel 1 Demographic data of the included studies.

\begin{tabular}{|c|c|c|c|c|c|c|}
\hline $\begin{array}{l}N \\
0\end{array}$ & Author & Article title & Year & Purpose & $\begin{array}{l}\text { No of teeth } \\
\text { (per group) }\end{array}$ & $\begin{array}{l}\text { Type of } \\
\text { Teeth }\end{array}$ \\
\hline & $\begin{array}{l}\text { Schmi- } \\
\text { dlin PR }{ }^{11}\end{array}$ & $\begin{array}{l}\text { Fracture resistance of endo- } \\
\text { dontically treated teeth with- } \\
\text { out ferrule using a novel H- } \\
\text { shaped short post }\end{array}$ & 2015 & $\begin{array}{l}\text { Evaluate the fracture resistance and fail- } \\
\text { ure type of modified } \mathrm{H} \text {-designed intradent- } \\
\text { al short retention preparation for } \mathrm{CAD} / \\
\text { CAM restorations, in cases where no fer- } \\
\text { rule is possible. }\end{array}$ & $40(10)$ & $\begin{array}{l}\text { Premolar } \\
\text { (single } \\
\text { root) }\end{array}$ \\
\hline 2 & Guo $J^{12}$ & $\begin{array}{l}\text { A comparison of the fracture } \\
\text { resistance of endodontically } \\
\text { treated lower premolars res- } \\
\text { tored with endocrowns and } \\
\text { glass fiberpost-core retained } \\
\text { conventional crowns }\end{array}$ & 2016 & $\begin{array}{l}\text { Evaluate the fracture resistances and } \\
\text { failure modes of endodontically treated } \\
\text { lower premolars restored with endo- } \\
\text { crowns and conventional post-core retain- } \\
\text { ed crowns }\end{array}$ & $30(10)$ & $\begin{array}{l}\text { Lower } \\
\text { Premolar }\end{array}$ \\
\hline 3 & Atash $^{8}$ & $\begin{array}{l}\text { Comparison of resistance to } \\
\text { fracture between } 3 \text { types of } \\
\text { permanentrestorations sub- } \\
\text { jected to shear force: An in } \\
\text { vitro study }\end{array}$ & 2017 & $\begin{array}{l}\text { Compare resistance to fracture between } \\
\text { endocrown and conventional post and } \\
\text { core restorations when subjected to shear } \\
\text { force. }\end{array}$ & $30(10)$ & $\begin{array}{l}\text { Lower } \\
\text { Premolar }\end{array}$ \\
\hline & $\begin{array}{l}\text { Koglu } \\
\mathrm{GM}^{7}\end{array}$ & $\begin{array}{l}\text { Fracture strength of CAD/ } \\
\text { CAM fabricated lithium disili- } \\
\text { cate and resin nano ceramic } \\
\text { restorations used for endo- } \\
\text { dontically treated teeth }\end{array}$ & 2017 & $\begin{array}{l}\text { Evaluate and compare fracture strength } \\
\text { and failure modes of endocrowns, zircon- } \\
\text { nia post, and fiber post supported resto- } \\
\text { rations and predict the clinical outcomes } \\
\text { of six different prostheses used for endo- } \\
\text { dontically treated teeth. }\end{array}$ & $60(10)$ & $\begin{array}{l}\text { Upper } \\
\text { central } \\
\text { incisor }\end{array}$ \\
\hline 5 & $\begin{array}{l}\text { de } \\
\text { Kuijper } \\
\mathrm{M}^{13}\end{array}$ & $\begin{array}{l}\text { Fracture strength of various } \\
\text { types of large direct compo- } \\
\text { site and indirect glass cera- } \\
\text { mic restorations }\end{array}$ & 2019 & $\begin{array}{l}\text { Investigate the mechanical behavior of } \\
\text { severely compromised endodontically } \\
\text { treated molars restored by means of va- } \\
\text { rious types of composite buildups, full- } \\
\text { contour lithium disilicate crowns (with or } \\
\text { without post) or a lithium disilicate endo- } \\
\text { crown }\end{array}$ & $105(15)$ & $\begin{array}{l}\text { Third } \\
\text { Molar }\end{array}$ \\
\hline 6 & $\begin{array}{l}\text { Alghala- } \\
\text { yini } S^{9}\end{array}$ & $\begin{array}{l}\text { Fracture load of nano-cera- } \\
\text { mic composite material for } \\
\text { anterior endocrown resto- } \\
\text { rations }\end{array}$ & 2020 & $\begin{array}{l}\text { Evaluate ability of nano ceramic compo- } \\
\text { site endocrown to withstand occlusal } \\
\text { forces when used in the anterior region }\end{array}$ & $80(10)$ & $\begin{array}{l}\text { Upper } \\
\text { central } \\
\text { incisor }\end{array}$ \\
\hline & $\begin{array}{l}\text { Sedrez- } \\
\text { Porto } \\
J^{10}\end{array}$ & $\begin{array}{l}\text { Which materials would acc- } \\
\text { ountforabetter mechanical } \\
\text { behavior for direct endo- } \\
\text { crown restorations }\end{array}$ & 2020 & $\begin{array}{l}\text { Investigate the mechanical performance } \\
\text { and fracture behavior of endocrown res- } \\
\text { toration prepared using different compo- } \\
\text { site materials and following a direct } \\
\text { technique. }\end{array}$ & $63(7)$ & $\begin{array}{l}\text { Lower } \\
\text { First } \\
\text { Molar }\end{array}$ \\
\hline
\end{tabular}

\section{DISCUSSION}

The goal of tooth restoration after endodontic treatment is to restore the function of the tooth. As another goal is to maintain the tooth and its restoration as long as possible. In some cases, after endodontic treatment tooth will require post-core crown restorative treatment. However, based on recent research, the use of post does notguarantee durability of the tooth. Root fracture and leakage are two things that can make a failure in the restoration with a post. Metal post are considered too rigid so they can make the tooth become fractured. ${ }^{7}$ An alternative option is to use fiber post. These fiber post are assessed to have a lower modulus of elasticity than the metal post and resemble dentin. It is expected to have a better distribution of masticatory forces and fracture resistance. ${ }^{7,12}$ But apparently both types of post are still unable to maintain teeth after endodontic treatment. According to Atash, et al. the failure of a metal post is centered on the risk of an unfavorable fracture, that is a fracture that makes the root unmanageable. ${ }^{8}$ In contrast to metal post, failures that occur in teeth with fiber post are more focused on crown restoration, so it can be said to be more beneficial. However, this does not rule out the possibility that fiber post can cause root fractures, even with a smaller percentage of probability. ${ }^{8}$ Based on studies conducted by Magne, post is considered to weaken the structure of the tooth, especially the rootcanal, making fracture vulnerable. ${ }^{14}$ 
Tabel 2 Groups evaluated with fracture strength (N) and standard deviation (SD).

\begin{tabular}{|c|c|c|c|c|c|}
\hline & Author & $\begin{array}{l}\text { Testing } \\
\text { methods }\end{array}$ & Groups & Materials & $\begin{array}{l}\text { Fracture strength } \\
\text { (N) Mean }( \pm S D)\end{array}$ \\
\hline 1 & $\begin{array}{l}\text { Schmidlin } \\
\text { PR }\end{array}$ & $\begin{array}{l}\text { UTM with a } \\
5 \mathrm{~mm} \text { steel } \\
\text { sphere at } \\
30^{\circ} \text { and a } \\
\text { cross-head } \\
\text { speed of } 1 \\
\mathrm{~mm} / \mathrm{min}\end{array}$ & $\begin{array}{l}\text { 1. H-post (glass-ceramic) } \\
\text { 2. H-post (LiDi) } \\
\text { 3. Endrocrown } \\
\text { 4. Control (Fiber post }+2 \\
\text { mm ferrule) }\end{array}$ & $\begin{array}{l}\text { 1. Glass-ceramic crown (IPS } \\
\text { Empress CAD) } \\
\text { 2. Lithium disilicate ceramic } \\
\text { (e.max CAD) } \\
\text { 3. Glass-ceramic crown } \\
\text { 4. Fiberpost + Glass-ceramic } \\
\text { crown }\end{array}$ & $\begin{array}{l}1.547 \pm 232 \\
2.1044 \pm 501 \\
3.592 .4 \pm 147 \\
4.890 \pm 125\end{array}$ \\
\hline 2 & Guo J & $\begin{array}{l}\text { Load test } \\
\text { with UTM a } \\
5 \mathrm{mmsteel} \\
\text { sphere at } \\
45^{\circ} \text { and a } \\
\text { cross-head } \\
\text { speed of } 1 \\
\mathrm{~mm} / \mathrm{min}\end{array}$ & $\begin{array}{l}\text { 1. Intact teeth (GI) } \\
\text { 2. Endocrown (GE) } \\
\text { 3. Conventional post-core } \\
\text { sup-ported crown group } \\
\text { (GC) }\end{array}$ & $\begin{array}{l}\text { 1. - } \\
\text { 2. IPS e.max CAD (Lithium } \\
\text { disilicate) } \\
\text { 3. RTD Post \#1.2 + IPS e.max } \\
\text { CAD }\end{array}$ & $\begin{array}{l}1.997 .1 \pm 166.3 \\
2.479 .1 \pm 180.6 \\
3.510 .1 \pm 191.0\end{array}$ \\
\hline 3 & Atash $\mathrm{R}$ & $\begin{array}{l}\text { Instron } 5585 \\
\text { test } \\
\text { machine } \\
\text { and a cross- } \\
\text { head speed } \\
\text { of } 1 \mathrm{~mm} / \mathrm{min}\end{array}$ & $\begin{array}{l}\text { 1. All ceramic endocrown } \\
\text { 2. Glass fiber post }+ \\
\text { composite resin core }+ \\
\text { ceramic crown } \\
\text { 3. cast post and core }+ \\
\text { ceramic crown }\end{array}$ & $\begin{array}{l}\text { 1. Ceramic crown (IPS e.max) } \\
\text { 2. Glass fiber post ( } 3 \mathrm{M} \\
\text { ESPE)+Com-posite resin } \\
\text { core (3M ESPE Filtek } \\
\text { Supreme XTE) + ceramic } \\
\text { crown } \\
\text { 3. Non-precious metal } \\
\text { (Wirobond C Co-Cr) + } \\
\text { ceramic crown }\end{array}$ & $\begin{array}{l}1.1717 .17 \pm 481.13 \\
2.1091 .11 \pm 179.03 \\
3.1068 .82 \pm 201.90\end{array}$ \\
\hline$\overline{4}$ & $\begin{array}{l}\text { KOĞLU } \\
\text { GM }\end{array}$ & $\begin{array}{l}\text { UTM with a } \\
2 \mathrm{~mm} \text { steel } \\
\text { sphere at } \\
45^{\circ} \text { and a } \\
\text { cross-head } \\
\text { speed of } 1 \\
\mathrm{~mm} / \mathrm{min}\end{array}$ & $\begin{array}{l}\text { 1. zirconia post/resinnano- } \\
\text { ceramic crown (ZrRNC) } \\
\text { 2. fiber post/resinnano- } \\
\text { ceramic crown (FbRNC) } \\
\text { 3. zirconia post/lithium } \\
\text { disilicate ceramic crown } \\
\text { (ZrLDS) } \\
\text { 4. fiber post/lithium disili- } \\
\text { cate ceramic crown } \\
\text { (FbLDS) } \\
\text { 5. resin-nano-ceramic } \\
\text { endocrown (EndoRNC) } \\
\text { 6. lithium disilicate } \\
\text { ceramic endocrown } \\
\text { (EndoLDS) }\end{array}$ & $\begin{array}{l}\text { 1. Zirconia post (IncorisTZI) + } \\
\text { Resin nano ceramic (Lava } \\
\text { Ultimate, 3M ESPE) } \\
\text { 2. Glass fiber posts (Ika-Dent, } \\
\text { Kutno, Poland) + Resin } \\
\text { nano ceramic } \\
\text { 3. Zirconia post + Lithium } \\
\text { disilicate (IPS e.max CAD, } \\
\text { Ivoclar Vivadent) } \\
\text { 4. Glass fiber post + LiDi } \\
\text { 5. Resin nano ceramic } \\
\text { 6. LiDi }\end{array}$ & $\begin{array}{l}1.893 .43 \\
2.764 .63 \\
3.580 .02 \\
4.646 .78 \\
5.869 .04 \\
6.915 .91\end{array}$ \\
\hline 5 & $\begin{array}{l}\text { de Kuijper } \\
M\end{array}$ & $\begin{array}{l}\text { Fracture } \\
\text { test: loaded } \\
\text { using } 8 \mathrm{~mm} \\
\text { ball-shaped } \\
\text { at oclusal } \\
\text { plane }(1 \\
\mathrm{mm} / \mathrm{min})\end{array}$ & $\begin{array}{l}\text { 1. Control (no prepn) } \\
\text { 2. Glass fiber reinforced } \\
\text { composite (GFRC) } \\
\text { 3. Microhybrid composite } \\
\text { (C) } \\
\text { 4. Microhybrid Composite } \\
\text { + post (CP) } \\
\text { 5. Lithium disilicate full } \\
\text { contour crown (LDS) } \\
\text { 6. Lithium disilicate full } \\
\text { contour crown and } \\
\text { glass fiber post (P-LDS) } \\
\text { 7. Endocrown (EC) }\end{array}$ & $\begin{array}{l}\text { 1. - } \\
\text { 2. Microhybrid composite (GC } \\
\text { Essentia Universal )+ GC } \\
\text { Ever X Posterior at central } \\
\text { pulp } \\
\text { 3. Composite resin (Clearfil } \\
\text { AP-X Posterior) } \\
\text { 4. Fiber post (Rely X Fiber } \\
\text { post red) + Core buildup } \\
\text { (Clearfil FC Core Plus } \\
\text { Dentin) + Composite resin } \\
\text { 5. Composite resin + IPS } \\
\text { e.max CAD } \\
\text { 6. Fiber post + Core buildup + } \\
\text { IPS e.max CAD } \\
\text { 7. IPS e.max CAD }\end{array}$ & $\begin{array}{l}4.1830 \pm 590 \\
5.3217 \pm 1052 \\
6.2694 \pm 665 \\
7.2425 \pm 993\end{array}$ \\
\hline
\end{tabular}


(cont) Tabel 2 Groups evaluated with fracture strength (N) and standard deviation (SD).

\begin{tabular}{|c|c|c|c|c|c|}
\hline & Author & $\begin{array}{l}\text { Testing } \\
\text { methods }\end{array}$ & Groups & Materials & $\begin{array}{l}\text { Fracture strength } \\
\text { (N) Mean }( \pm S D)\end{array}$ \\
\hline 6 & $\begin{array}{l}\text { Alghala- } \\
\text { yini S. }\end{array}$ & $\begin{array}{l}\text { Compressive } \\
\text { static load with } \\
\text { load on the } \\
\text { palatal surface } \\
\text { just above the } \\
\text { cingulum at a } \\
130^{\circ} \text { and a } \\
\text { cross-head } \\
\text { speed of } 1 \\
\mathrm{~mm} / \mathrm{min}\end{array}$ & $\begin{array}{l}\text { Post, core and crown } \\
\text { restoration (control) } \\
\text { 1. IPS e.max } 0.5 \mathrm{~mm} \text { above } \\
\text { CEJ } \\
\text { 2. IPS e.max } 2 \mathrm{~mm} \text { above } \\
\text { CEJ } \\
\text { 3. Lava Ultimate } 0.5 \mathrm{~mm} \\
\text { above CEJ } \\
\text { 4. Lava Ultimate } 2 \mathrm{~mm} \\
\text { above CEJ } \\
\text { Endocrown restoration } \\
\text { 5. IPS e.max } 0.5 \mathrm{~mm} \text { above } \\
\text { CEJ } \\
\text { 6. IPS e.max } 2 \mathrm{~mm} \text { above } \\
\text { CEJ } \\
\text { 7. Lava Ultimate } 0.5 \mathrm{~mm} \\
\text { above CEJ } \\
\text { 8. Lava Ultimate } 2 \mathrm{~mm} \\
\text { above CEJ }\end{array}$ & $\begin{array}{l}\text { Post: RelyX Fiber Post (3M } \\
\text { ESPE) } \\
\text { Crown: IPS e.max (Ivoclar) / } \\
\text { Lava Ultimate (3M ESPE) }\end{array}$ & $\begin{array}{l}1.627 .9 \\
2.449 .1 \\
3.1073 .8 \\
4.1019 .6 \\
5.667 .2 \\
6.421 .7 \\
7.1130 .8 \\
8.1119 .1\end{array}$ \\
\hline 7 & $\begin{array}{l}\text { Sedrez- } \\
\text { Porto } \\
\text { JA }\end{array}$ & $\begin{array}{l}\text { Universal } \\
\text { testing } \\
\text { machine, } \\
\text { cross-head } \\
\text { speed of } 1 \\
\mathrm{~mm} / \mathrm{min}\end{array}$ & $\begin{array}{ll}\text { 1. } & \text { Control (sound tooth) } \\
2 . & \text { Endocrown (E.max) } \\
\text { 3. } & \text { Endocrown (Z350) } \\
\text { 4. } & \text { Endocrown } \\
\text { (Z350_SBMP) } \\
\text { 5. } & \text { Endocrown } \\
& \text { (Z350_SBU) } \\
\text { 6. } & \text { Endocrown (Bulk-Fill) } \\
\text { 7. } & \text { Post-retained } \\
\text { restoration (GFP_Z350) } \\
\text { 8. } & \text { Post-retained } \\
& \text { restoration } \\
& \text { (GFP_Z350_SBMP) } \\
\text { 9. } & \text { Post-retained } \\
& \text { restoration } \\
& \text { (GFP_Bulk_Fill) }\end{array}$ & $\begin{array}{l}\text { 1. - } \\
\text { 2. IPS e.max lithium disilicate } \\
\text { 3. Conventional resin compo- } \\
\text { site (Filtek }^{\mathrm{TM}} \mathrm{Z} 350 \mathrm{XT} \text { ) } \\
\text { 4. Filtek } \\
\text { (Scotch Z350 XT + SBMP } \\
\text { Purpose Adhesive) } \\
\text { 5. Filtek }{ }^{\mathrm{TM}} \mathrm{Z} 350 \mathrm{XT}+\mathrm{SBU} \\
\text { (Scotchbond }^{\mathrm{TM}} \text { Universal } \\
\text { Adhesive) } \\
\text { 6. Filtek }{ }^{\mathrm{TM}} \text { Bulk Fill } \\
\text { 7. White Post DC + Filtek }{ }^{\mathrm{TM}} \\
\text { Z350 XT } \\
\text { 8. White Post DC + Filtek } \\
\text { Z350 XT + SBMP } \\
\text { 9. White Post DC + Filtek } \\
\text { Bulk Fill }\end{array}$ & $\begin{array}{l}\text { 1. } 2149.9 \pm 543.3 \\
\text { 2. } 1748.5 \pm 559.3 \\
\text { 3. } 2292.3 \pm 716.8 \\
\text { 4. } 2546.3 \pm 216.8 \\
\text { 5. } 2583.7 \pm 612.2 \\
\text { 6. } 3363.1 \pm 123.9 \\
\text { 7. } 2451.6 \pm 484.5 \\
\text { 8. } 2774.0 \pm 578.8 \\
\text { 9. } 2861.2 \pm 424.1\end{array}$ \\
\hline
\end{tabular}

Studies in vivo also said that it is better to have teeth with ferrules without post than teeth with post, but without ferrules. ${ }^{3}$ Types of post, either metal or fiber, have no significant difference in these studies. ${ }^{3}$ This shows that post is not mandatory afterendodontic post treatment tooth. Then the question arisen, if without a post, is it better to do a core build-up and then restore it with a crown? All the way through in-vitro research by Magne, core build-up cannot always maintain restoration and remaining teeth. The higher core build-up will result in a lower survival rate, based on this reason, Magne suggested the use of endocrown restoration. ${ }^{2}$ Endocrown is a restoration that is a single unit between the crown and the core. Although the literature on endocrown is still limited, empirical evidence leads to a positive point for the use of this restoration. Endocrown is known as a minimally invasive type of restoration, which maintains as many healthy tooth structure as possible. ${ }^{9}$ The success of endocrown is in the ability of adhesion of restoration material to the tooth surface. However, the use of appropriate materials can also affect the success of a restoration. ${ }^{13}$ Each material has a different modulus of elasticity, it is recommended that a good restoration material should have a modulus of elasticity close to enamel \& dentin. ${ }^{7}$ The closer the modulus of elasticity between the teeth and the restorative material, the more even the spread of the load. Composite resin material as an alternative in 
T abel 3 Groups evaluated with fracture type

\begin{tabular}{|c|c|c|c|c|c|}
\hline $\begin{array}{l}\mathrm{N} \\
\mathrm{O}\end{array}$ & Study & Groups & $\begin{array}{l}\text { Number of } \\
\text { favorable } \\
\text { fractures }\end{array}$ & $\begin{array}{c}\text { Number of } \\
\text { unfavorable } \\
\text { fractures }\end{array}$ & $\begin{array}{c}\text { Unfavorable Fracture } \\
\text { description }\end{array}$ \\
\hline 1 & $\begin{array}{l}\text { Schmidlin } \\
\text { PR }\end{array}$ & $\begin{array}{l}\text { a. H-post (glass-ceramic) } \\
\text { b. H-post (LiDi) } \\
\text { c. Endrocrown } \\
\text { a. Control (Fiber post }+2 \mathrm{~mm} \text { ferrule) }\end{array}$ & $\begin{array}{l}\text { a. } 90 \% \\
\text { b. } 70 \\
\text { c. } 100 \% \\
\text { d. } 50 \%\end{array}$ & $\begin{array}{l}\text { a. } 10 \% \\
\text { b. } 30 \% \\
\text { c. } 0 \% \\
\text { d. } 50 \%\end{array}$ & $\begin{array}{l}\text { Tooth/root fracture that } \\
\text { would necessitate tooth } \\
\text { extraction }\end{array}$ \\
\hline 2 & Atash $\mathrm{R}$ & $\begin{array}{l}\text { a. All ceramic endocrown } \\
\text { b. Glass fiber post + composite resin core } \\
\quad+\text { ceramic crown } \\
\text { b. cast post and core + ceramic crown }\end{array}$ & $\begin{array}{l}\text { a. } 3 \\
\text { b. } 6 \\
\text { c. } 1\end{array}$ & $\begin{array}{l}\text { a. } 7 \\
\text { b. } 4 \\
\text { c. } 9\end{array}$ & $\begin{array}{l}\text { a. } 2 \text { loosening } \\
\text { b. restoration fracture, } \\
\text { root unbroken } \\
\text { c. restoration fracture, } \\
\text { root unbroken }\end{array}$ \\
\hline 3 & Guo J & $\begin{array}{l}\text { a. Intact teeth (GI) } \\
\text { b. Endocrown (GE) } \\
\text { c. Conventional post-core supported } \\
\text { crown group (GC) }\end{array}$ & $\begin{array}{l}\text { a. } 7 \\
\text { b. } 4 \\
\text { c. } 4\end{array}$ & $\begin{array}{l}\text { a. } 3 \\
\text { b. } 6 \\
\text { c. } 6\end{array}$ & $\begin{array}{l}\text { Non-repairable fractures } \\
\text { below the level of bone } \\
\text { simulation }\end{array}$ \\
\hline 4 & $\begin{array}{l}\text { KOGGLU } \\
\text { GÜNGÖR, } \\
\text { Merve }\end{array}$ & $\begin{array}{l}\text { c. zirconia post/resin-nano-ceramic crown } \\
\text { (ZrRNC) } \\
\text { d. fiber post/resinnano-ceramic crown } \\
\text { (FbRNC) } \\
\text { e. zirconia post/lithium disilicate ceramic } \\
\text { crown (ZrLDS) } \\
\text { f. fiber post/lithium disilicate ceramic } \\
\text { crown (FbLDS) } \\
\text { g. resin-nano-ceramic endocrown } \\
\text { (EndoRNC) } \\
\text { d. lithium disilicate ceramic endocrown } \\
\text { (EndoLDS) }\end{array}$ & $\begin{array}{l}\text { a. } 10 \\
\text { b. } 10 \\
\text { c. } 10 \\
\text { d. } 10 \\
\text { e. } 0 \\
\text { d. } 3\end{array}$ & $\begin{array}{l}\text { a. } 0 \\
\text { b. } 0 \\
\text { c. } 0 \\
\text { d. } 0 \\
\text { e. } 10 \\
\text { d. } 7\end{array}$ & Root fracture \\
\hline 5 & $\begin{array}{l}\text { de Kuijper } \\
M\end{array}$ & $\begin{array}{l}\text { a. Control (no preparation) } \\
\text { b. Glass fiber reinforced composite } \\
\text { (GFRC) } \\
\text { c. Microhybrid composite (C) } \\
\text { d. Microhybrid Composite }+ \text { post (CP) } \\
\text { e. Lithium disilicate full contour crown } \\
\text { (LDS) } \\
\text { f. Lithium disilicate full contour crown and } \\
\text { glass fiber post (P-LDS) } \\
\text { g. Endocrown (EC) }\end{array}$ & $\begin{array}{l}\text { a. } 4 \\
\text { b. } 10 \\
\text { c. } 1 \\
\text { d. } 1 \\
\text { e. } 5 \\
\text { f. } 4 \\
\text { g. } 3\end{array}$ & $\begin{array}{l}\text { a. } 11 \\
\text { b. } 5 \\
\text { c. } 14 \\
\text { d. } 14 \\
\text { e. } 10 \\
\text { f. } 10 \\
\text { g. } 12\end{array}$ & $\begin{array}{l}\text { a. Fracture }>1 \mathrm{~mm} \text { below } \\
\text { CEJ 1; Root fracture } 10 \\
\text { b. Fracture }>1 \mathrm{~mm} \text { below } \\
\text { CEJ } 4 \text {; Root fracture } 1 \\
\text { c. Root fracture } 14 \\
\text { d. Fracture }>1 \mathrm{~mm} \text { below } \\
\text { CEJ 3; Root fracture } 11 \\
\text { e. Fracture }>1 \mathrm{~mm} \text { below } \\
\text { CEJ } 1 \text {; Root fracture } 9 \\
\text { f. Root fracture } 10 \\
\text { g. Fracture }>1 \mathrm{~mm} \text { below } \\
\text { CEJ 1; Root fracture } 11\end{array}$ \\
\hline 6 & $\begin{array}{l}\text { Alghalayini } \\
\text { S. }\end{array}$ & $\begin{array}{l}\text { Post, core and crown restoration (control) } \\
\text { a. IPS e.max } 0.5 \mathrm{~mm} \text { above CEJ } \\
\text { b. IPS e.max } 2 \mathrm{~mm} \text { above CEJ } \\
\text { c. Lava Ultimate } 0.5 \mathrm{~mm} \text { above CEJ } \\
\text { d. Lava Ultimate } 2 \mathrm{~mm} \text { above CEJ } \\
\text { Endocrown restoration } \\
\text { e. IPS e.max } 0.5 \mathrm{~mm} \text { above CEJ } \\
\text { f. IPS e.max } 2 \mathrm{~mm} \text { above CEJ } \\
\text { g. Lava Ultimate } 0.5 \mathrm{~mm} \text { above CEJ } \\
\text { c. Lava Ultimate } 2 \mathrm{~mm} \text { above CEJ }\end{array}$ & $\begin{array}{l}\text { a. } 80 \% \\
\text { b. } 40 \% \\
\text { c. } 60 \% \\
\text { d. } 0 \% \\
\text { e. } 60 \% \\
\text { f. } 100 \% \\
\text { g. } 80 \% \\
\text { c. } 40 \%\end{array}$ & $\begin{array}{ll}\text { a. } 20 \% \\
\text { b. } 60 \% \\
\text { c. } 40 \% \\
\text { d. } 100 \% \\
\text { e. } 40 \% \\
\text { f. } 0 \% \\
\text { g. } 20 \% \\
\text { d. } 60 \%\end{array}$ & $\begin{array}{l}\text { Fracture extend beyond } \\
\text { the cemento-enamel } \\
\text { junctions }\end{array}$ \\
\hline 7 & $\begin{array}{l}\text { Sedrez- } \\
\text { Porto JA }\end{array}$ & $\begin{array}{l}\text { a. Control (sound tooth) } \\
\text { b. Endocrown (E.max) } \\
\text { c. Endocrown (Z350) } \\
\text { d. Endocrown (Z350_SBMP) } \\
\text { e. Endocrown (Z350_SBU) } \\
\text { f. Endocrown (Bulk-Fill) } \\
\text { g. Post-retained restoration (GFP_Z350) } \\
\text { h. Post-retained restoration } \\
\quad \text { (GFP_Z350_SBMP) } \\
\text { i. Post-retained restoration(GFP Bulk Fill) }\end{array}$ & $\begin{array}{l}\text { a. } 85.7 \% \\
\text { b. } 28.6 \% \\
\text { c. } 28.6 \% \\
\text { d. } 71.4 \% \\
\text { e. } 42.9 \% \\
\text { f. } 71.4 \% \\
\text { g. } 14.3 \% \\
\text { h. } 42.9 \% \\
\text { i. } 28.6 \%\end{array}$ & $\begin{array}{l}\text { a. } 14.3 \% \\
\text { b. } 71.4 \% \\
\text { c. } 71.4 \% \\
\text { d. } 28.6 \% \\
\text { e. } 57.1 \% \\
\text { f. } 28.6 \% \\
\text { g. } 85.7 \% \\
\text { h. } 57.1 \% \\
\text { i. } 71.4 \%\end{array}$ & Root fracture \\
\hline
\end{tabular}


making endocrown crowns can be considered. According to Sedrez-Proto JA et al., endocrown crowns with composite resins have a mechanical ability that resembles conventional crown restorative materials such as glass-ceramics, but is better because the resin composite endocrown are considered to be able to protect the remaining tooth structure than e.max restoration materials. $^{10}$

When used for anterior teeth, endocrown has no better fracture resistance and post and crown restoration. ${ }^{13}$ The same finding for premolar teeth was also stated by Guo, et al. ${ }^{12}$ However, endocrown for anterior teeth produce more unfavorable fracture than post. ${ }^{13}$ This might be because endocrown is considered like a short post.

Answer the PICOquestion, according to most studies, tooth with root canal treatment restored with endocrown has better fracture resistance compared than post and crown. However, endocrown does not provide better protection against fractures that occurcompared to post and crown.

\section{REFERENCES}

1. Aurélio $M$, Carvalho $D$. Current options concerning the endodontically-treated teeth restoration with the adhesive approach Partial restorations versus full Preserving the coronal structure. 2018;32:147-158.

2. Ao PM, Bruzi CG. Influence of no-ferrule and no-post buildup design on the fatigue resistance of endodontically treated molars restored with resin nanoceramic CAD/CAM crowns. 2014:595-602. doi:10.2341/ 13-004-L

3. Naumann M, Schmitter M, Frankenberger R, Krastl G. "'Ferrule Comes First . Post Is Second!"' Fake News and Alternative Facts? A Systematic Review. J Endod. 2017. doi:10.1016/j.joen.2017.09.020

4. Einhorn M, Duvall N, Wajdowicz M, Brewster J, Roberts H. Preparation ferrule design effect on endocrown failure resistance. 2019:237-242. doi:10.1111/jopr.12671

5. Derleme E, Sevimli G, Cenglz S, Oruç MS. Endocrowns: review. 2015;49(2):57-63.

6. Sedrez-porto JA, Luiz W, Oliveira D, Fernandes A, Münchow EA, Pereira-cenci T. Endocrown restorations : A systematic review and meta-analysis. J Dent. 2016;52:8-14. doi:10.1016/j.jdent.2016.07.005

7. Güngör MB, Bal BT, Yilmaz H, Aydin C, Nemli SK. Fracture strength of CAD / CAM fabricated lithium disilicate and resin nano ceramic restorations used for endodontically treated teeth. 2017. doi:10.4012/dmj.2016-017

8. Atash R, Arab M, Duterme H, Cetik S. Comparison of resistance to fracture between three types of permanent restorations subjected to shear force : An in vitro study. 2017:239-249. doi:10.4103/jips.jips

9. Article O. Fracture load of nano-ceramic composite material for anterior endocrown restorations. 2020; 23 (1):1-9. doi:10.14295/bds.2020.v23i1.1853

10. Sedrez-porto A, Aldrighi E, Sergio M, Pereira-cenci T. J Mechanical Behavior of Biomedical Materials Which materials would account for a better mechanical behavior for direct endocrown restorations? 2020;103 (December 2019). doi:10.1016/j.jmbbm.2019.103592

11. Patrick R, Schmidlin PR, Fh D, Deabreu D. Restorative dentistry fracture resistance of endodontically treated teeth without ferrule using a novel H-shaped short post. 2015;46(2):97-109. doi:10.3290/j.qi.a32634

12. Guo J, Wang Z, Li X, Sun C, Gao E, Li H. A comparison of the fracture resistances of endodontically treated mandibular premolars restored with endocrowns and glass fiber post-core retained conventional crowns. 2016:489-93.

13. Haumahu D, Schepke U. Fracture strength of various types of large direct composite and indirect glass ceramic restorations. doi:10.2341/18-111-L

14. Magne P, Lazari PC, Carvalho MA, Johnson T DBCA. Ferrule-effect dominates over use of a fiber post when restoring endodontically treated incisors : an in vitro study. Oper Dent. 2017;42:396-406. doi:10.2341/ 16-243-L 